\title{
Vom Exil- zum Diaspora-Islam. Muslimische Identitäten in Europa ${ }^{1}$
}

\section{Von Werner Schiffauer}

Die Muslime in Europa stehen vor der Herausforderung, eine Rolle für den Islam außerhalb der klassischen islamischen Länder, des dar al Islam, zu definieren. Dies bedeutet, sich als Muslime in dreierlei Hinsicht neu zu verorten - in Bezug auf das Einwandererland, in Bezug auf das Herkunftsland und in Bezug auf den globalen Islam.

Die Verortung als Muslim in der Einwanderergesellschaft im Besonderen und in Europa im Allgemeinen wird durch zwei Besonderheiten erschwert: Zum einen gibt es eine lange (und von beiden Seiten immer wieder gepflegte) Tradition, Islam und Christen in einer Beziehung von Alterität zu sehen, d.h. ein vorgeblich »islamisches« und ein vorgeblich »christlich-jüdisches« Wertverständnis in einen Antagonismus zueinander zu setzen. Zum anderen sind die Trägerschichten des Islam zum größten Teil Arbeitsmigranten und ihre Abkömmlinge. Es handelt sich um Neuankömmlinge, die in Europa die unteren Positionen der Berufsskala eingenommen haben und sich über die Generationen langsam hocharbeiten. Der Islam ist somit nicht nur die andere Religion schlechthin, sie ist auch die Religion der Arbeiter, der underclass, der Ausgegrenzten und der Ghettobewohner. Diese beiden Aspekte unterscheiden die Situation des Islam in Europa von der in anderen Regionen, in der der Islam in der Minderheit steht.

Die Muslime müssen zweitens den Bezug zum Herkunftsland neu denken. Die durch eine geteilte Lebenswelt gestifteten Selbstverständlichkeiten verlieren ihre Plausibilität. Man betrachtet das Herkunftsland (und die Rolle, die die Religion in ihm spielt) von außen, auf der Folie und in der Differenz zur Einwanderungsgesellschaft.

Zum dritten entfaltet sich ein neuer Bezug zum Islam als Weltreligion und damit zur um$m a$, der islamischen Weltgemeinschaft. Zumindest in den Ländern des Nahen und Mittleren Ostens existiert im Alltagsverständnis eine ethnozentrisch bedingte und wenig reflektierte Identifikation von Nation und Islam. Man weiß natürlich, dass außerhalb der arabischen Nation respektive türkischen Nation Muslime leben, aber im Grund ist Araber-Sein und MuslimSein respektive Türke-Sein und Muslim-Sein identisch. ${ }^{2}$ Auch diese Identifikation bricht mit der Migration auf. In Europa wird man als Muslim identifiziert und für die Ereignisse in der islamischen Welt haftbar gemacht. Man muss sich zu Körperstrafen, Burka und dem 11. September erklären, auch wenn diese Phänomene für die eigene islamische Praxis und die der Umwelt nie eine Rolle spielten.

Es entspricht dieser Vielfalt der Bezüge, dass der sich in Europa etablierende Islam äuBerst vielfältig erscheint. Die Versuche sich zu verorten brachten eine Vielzahl von Antworten hervor. Anders als das Klischee es will, entsteht nicht ein, vorgeblich liberaler »europäischer Islam«, sondern der Islam in Europa ist durch gegensätzliche Strömungen und

1) Ich möchte an dieser Stelle meinen Dank an die vielen Personen ausrichten, die mit mir den Text kritisch durchgegangen sind und ihn kritisiert haben. Zu nennen sind zunächst meine Frau Julia Eckert und die Teilnehmer des Forschungskolloquiums in Frankfurt/Oder. Besonders hervorheben möchte ich auch den Beitrag der anonymen Rezensenten, deren Kritik mir sehr wichtig war.

2) In dieser Hinsicht unterscheidet sich das alltägliche Bewusstsein in den islamischen Ländern nicht von der Situation im Christentum. Die Identifikation von Christentum und Nation spiegelte sich in Europa besonders deutlich in der Militärgeschichte etwa in dem Brauch, die Waffen auch in Kriegen der christlichen Nationen untereinander zu segnen. 
Richtungen charakterisiert. Allerdings gilt es über die übliche (und mittlerweile etwas langweilige) Konstatierung von pluraler Identität oder Multivokalität hinaus und einen Schritt weiter zu gehen. Die Vielfalt der Stimmen und Positionen, die sich entwickeln, stehen nämlich nicht unverbunden nebeneinander, sondern sie beziehen sich aufeinander, ergänzen oder widersprechen sich. Sie können sich schon deshalb nicht gegenseitig ignorieren, weil sie, worauf Zygmunt Bauman hingewiesen hat, seitens der Mehrheitsgesellschaft als Kollektivperson, als Zurechnungsgemeinschaft, betrachtet werden (Bauman 1991: 42). Sie werden füreinander verantwortlich gemacht. Weil die Äußerungen oder Taten einzelner islamischer Gemeinden auf alle anderen Muslime zurückzufallen drohen, müssen sie Stellung beziehen und sich gegebenenfalls distanzieren. Dies ist besonders deutlich bei extremen Vorkommnissen wie der Bücherverbrennung in Bradford oder dem 11. September, gilt aber auch in weniger dramatischen Fällen. Weil dies so ist, konstituieren diese vielen Stimmen einen Raum von Debatten, eine Diskursarena oder ein Diskursfeld. Diskursfelder sind durch strittige Themen und eine daraus abgeleitete Konstellation von Positionen charakterisiert.

In diesem Text geht es darum, am Beispiel der türkischen Einwanderer die Vielfalt und die Gemeinsamkeit der Debatten über die Verortung des Islam in Europa zu rekonstruieren. Die Debatte der ersten Generation, so die These, zentrierte sich um andere Punkte, hatte andere Themen und führte zu anderen Gruppenkonstellationen als die Debatte der zweiten Generation. Für die erste Generation stellte sich das Problem, einen »Islam im Exil« zu definieren. Europa war für sie »gurbet«, Fremde. Sie fand sich in einer ex-zentrischen Position, die sie zwang, bewusster als im Heimatland zu dem islamischen kulturellen Repertoire Stellung zu beziehen und die es ihr ermöglichte, einen regard de loin auf ihr Heimatland zu richten. ${ }^{3}$ Die Fraktionen, die sich in dieser Generation herausbildeten, reflektierten die Rolle, die der Islam in der Türkei spielen sollte. Wenn dieser Islam auch türkeibezogen war, so unterschied er sich doch deutlich vom türkischen Islam, vor allem was die pointierte Schärfe betraf, mit der die Fraktionen einander gegenübertraten. Die Debatten in der zweiten Generation stellen sich dagegen der Notwendigkeit, in »den Kulturen, in denen sie leben, zurechtzukommen, ohne sich einfach zu assimilieren und ihre eigene Identität vollständig zu verlieren « (Hall 1992:310). Der Islam dieser Generation lässt sich als diasporischer Islam charakterisieren, insofern diese Generation in Europa heimisch ist, aber das starke Gefühl von transnationalen Zusammengehörigkeiten (etwa zu Türken in anderen europäischen Ländern und in der Türkei) pflegt. Dem korrespondiert ein größeres Bewusstsein von der umma, der islamischen Weltgemeinschaft. ${ }^{4}$ Ich möchte im Folgenden zeigen, dass in der Auseinandersetzung mit dem Anerkennungsproblem in der Aufnahmegesellschaft bei dieser Generation ähnliche Fraktionierungen entstehen, wie sie auch für andere Diasporagemeinden typisch sind - nämlich Flügel von einer Ultraorthodoxie, einer Orthodoxie und einer wertepluralen Position. Hier liegt eine meiner Differenzen zu Autoren wie Hall, Bhabha, Clifford oder Gilroy, denen ich, wie deutlich werden wird, ansonsten viel verdanke. Sie sehen in Hybridität den Kern ei-

3) Die von einer ersten Generation zu erbringenden Definitionsleistungen werden oft unterschätzt. Siehe in diesem Zusammenhang die Fallstudien von Yasșar Fuad und Fatma Eren in Schiffauer 1997.

4) Es bedarf noch einiger Anmerkungen zu der Unterscheidung zwischen Exil-Islam und Diaspora-Islam, da aus der jüdischen Religionsgeschichte stammende Begriff Diaspora zwar wörtlich »Ausbreitung « meint, semantisch aber am besten als Exil zu fassen ist. In diesem Text bezieht sich der Begriff Exil auf das existenzielle Gefühl, in der Fremde zu leben. Ihm korrespondiert das Gefühl des Verlustes der Heimat und eine entsprechende Sehnsucht nach Rückkehr. Diaspora bezieht sich dagegen auf das vor allem bei einer zweiten Generation auftretende Gefühl einer Alterität in einer neuen Heimat. Die hier vorgenommene Unterscheidung von exilischer und diasporischer Identität knüpft an die Überlegungen von Daniel und Jonathan Boyarin zur jüdischen Diaspora (1993) an. Sie argumentieren, dass jüdische Identität nur als Diaspora-Identität denkbar ist und nicht als abgeschlossenes, begrenztes Phänomen (1993:721) (siehe dazu auch Clifford 1994:323. Das Argument der Boyarins wurde von Philip Roth in seinem Roman Operation Shylock aufgenommen). 
ner Diaspora-Identität und werten ethnischen oder religiösen Fundamentalismus als bedauerliche Entgleisung. Ich betrachte dagegen gerade die fast notwendige Koexistenz dieser Flügel als Charakteristikum von Diaspora-Identität.

Theoretisch ist es mein Anliegen, die wichtigen Einsichten, die zum Verhältnis von Macht und Identität in der postkolonialen Diskussion entwickelt wurden, für die Diskussion der Entwicklung des europäischen Islam der zweiten und dritten Generation von Einwanderern fruchtbar zu machen. Dies bedeutet mehr als die Anwendung einer Theorie auf ein neues Gebiet. Der Islam ist nämlich nicht aus Zufall in der Diaspora-Diskussion bislang stiefmütterlich behandelt worden. Diese Vernachlässigung steht in Zusammenhang mit einer häufigen Vermengung normativer und empirischer Gehalte, die ihrerseits eine Folge der politischen Emphase der Theorie ist. Die von Brüchen, komplexen Verwerfungen, Zerstreuungen, Ausgrenzungen etc. charakterisierte Diaspora-Situation schien für viele postkoloniale Theoretiker die Chance zu bergen, die Fallen der Subjektzentriertheit zu überwinden, die Foucault herausgearbeitet hat. Die Diaspora bot Raum für eine kreative, kosmopolitische Existenz und damit eine Chance, sich zu emanzipieren. Die afro-karibische Diaspora galt dabei als das Lieblingskind: Sie brachte Formen des Protestes hervor, in denen sich die europäische Linke wiedererkennen konnte, weil sie ihr so nahe standen. In sie konnte man hineinprojizieren, wofür man sich aus einer linksrevolutionären Perspektive begeistern konnte. Die Folge war ein systematischer normativer bias der postkolonialen Theorie. Dieser bias tritt aus der Behandlung der Kultur, die sich unter den muslimischen Einwanderern entfaltete, deutlich hervor. Diese Diaspora-Kultur war nicht weniger radikal als die der karibischen Einwanderer. Aber es war deutlich, dass die islamischen Formen des Protestes nicht diejenigen waren, die eine europäische Linke mit Emanzipation assoziierte. Beispielhaft lässt sich dies in Bezug auf die Rolle, die der Inszenierung von Körperlichkeit in der Protestkultur nach Paul Gilroy zukommt, belegen: »The body, « schreibt Gilroy, »has become, in various ways, a cultural locus of resistance and desires. A sense of the body's place in the natural world can provide, for example, a social ecology and an alternative rationality that articulates a cultural and moral challenge to the exploitation and domination of the snature within us and without us<.«(1993: 407). All dies lässt sich sehr genau auf die Kopftuchbewegung (politics of veiling) beziehen. Dennoch war es eindeutig nicht diese Form von Körperpolitik, über die Gilroy sich mit solcher Begeisterung äußerte.

Die Hinwendung zu islamischen Formen des Protestes kann helfen, den normativen bias der postkolonialen Theorie zu überwinden. Der kulturvergleichende Blick erlaubt Distanz und stiftet eine gewisse Nüchternheit. Er öffnet damit den Blick für Empirie: Er kann dazu beitragen, die Gemeinsamkeit und Differenzen der Träume von Befreiung zu sehen, und macht es möglich, die Verschiedenartigkeit dieser Träume zu beschreiben. Er sensibilisiert für die Tatsache, dass die Träume der Befreiung der einen Gruppe nicht unbedingt identisch sind mit den Träumen der Befreiung einer anderen Gruppe - was soweit gehen kann, dass das, was im Namen der Befreiung von kultureller Fremdbestimmung etwa von Islamisten gefordert wird ${ }^{5}$, für andere, die Befreiung mit einer Bewegung zu mehr individueller Autonomie und Selbstbestimmung assoziiert, als Alptraum erscheinen kann. ${ }^{6}$

5) Zu diesen Träumen im Islamismus siehe Sivan 1985:1-15

6) Ich halte es für wichtig, den Begriff der »Befreiung« relativistisch zu bestimmen - auch wenn man sich damit das Problem einhandelt, dass der Begriff in einem islamischen Diskurs andere Konnotationen enthält als in einem antirassistischen Diskurs. Zum einen kann auch innerhalb eines Diskursfeldes der Begriff schon sehr unterschiedlich besetzt sein - eine radikal-feministische Bestimmung kann sich unterscheiden von einem antirassistischen oder indigenistischen (was beispielsweise bei den Zaptistas zu Problemen führte). Zum anderen trägt eine Relativierung der Binnensicht Rechnung und beugt einem Alterierungsdiskurs vor. 
Ich beziehe mich im Folgenden auf Material, das ich in mehr als zwanzig Jahren vor allem in der Auseinandersetzung mit der türkischen Diaspora in Deutschland erhoben habe. Eine in der letzten Zeit fertiggestellte vergleichende Untersuchung (Schiffauer et al. 2002) zeigt, dass sich zahlreiche Einsichten mit gewissen Einschränkungen auch auf die anderen europäischen Länder übertragen lassen.

\section{Der Islam im Exil}

Kollektive Identität entfaltet sich im Zusammenspiel von kollektiver Erfahrung und der politischen Organisation dieser Erfahrung. Erfahrung begründet eine - nicht selten diffuse - Bedürfnislage. Diese wird allerdings erst dann zu einem bewussten Selbstverhältnis, wenn sie ausgedrückt bzw. »repräsentiert« wird - und zwar in dem Doppelsinn des Wortes, wenn sie »dargestellt« und politisch vertreten wird. Dies geschieht manchmal »bottom up«, wenn etwa soziale Bewegungen ein Bedürfnis aufgreifen und ihm Ausdruck verleihen. Genauso häufig geschieht dieser Prozess »top down«: Dann reagieren bereits existierende Organisationen auf ein Bedürfnis und formulieren ein Deutungsangebot. In diesem Fall entsteht ein Verhältnis von Nachfrage und Angebot. Dies ist regelmäßig dann der Fall, wenn nicht nur eine Organisation, sondern eine Vielzahl von Organisationen auftritt, die mit ihren Deutungsangeboten untereinander konkurrieren.

Der Schlüsselbegriff zum Verständnis der religiösen Bedürfnislage bei Migranten der ersten Generation lautet »gurbet«, die Fremde. ${ }^{7}$ Die Erfahrung von Fremdheit hat mehrere Facetten. Eine erste ist die Angst vor Selbstverlust. Der Migrant bewegt sich, oft als Alleinstehender, in einem Raum, in dem ihn niemand kennt. Oft gewöhnt an ein hohes Ausmaß von sozialer Kontrolle findet er sich plötzlich in einem Feld, in dem soziale Kontrolle so gut wie nicht existiert. Dies führte oft zum Gefühl von Haltlosigkeit. Unter den Migranten der ersten Generation zirkulierten Erzählungen von türkischen Arbeitern, die in Deutschland »unter die Räder kamen«, Beziehungen zu Frauen aufnahmen, Alkoholiker wurden und darüber ihre Lebensperspektive opferten. Der Islam bot einen gewissen Halt gegen diese tendenziell sinnbedrohenden Erfahrungen - nicht zuletzt, weil man sich in einer Gemeinde von Gleichgesinnten aufhielt, die sich gegenseitig stützen konnten. Eine zweite Facette der Fremdheitserfahrung war die Konfrontation mit einer Sinnkrise. Wohl jeder Migrant stellt sich irgendwann die Frage, was er eigentlich in der als leidvoll empfundenen Fremde verloren hatte, ob er nicht besser zu Hause geblieben wäre. Die türkischen Migranten drückten dies oft mit der Metapher der Kälte aus. Auch hier hilft eine religiöse Orientierung, diese Frage zumindest besser zu bewältigen, wenn vielleicht auch nicht, sie zu beantworten. Auch bietet eine Gemeinde einen gewisse »Wärme«. Ein dritter und wohl der wesentlichste Aspekt des Erlebnisses von Fremdheit hing mit dem zu Beginn der siebziger Jahre einsetzenden Familiennachzug zusammen. Er bedeutete zum einen, dass man sich nun auf einen längeren Aufenthalt in Europa einrichtete. Er bedeutete zum anderen, dass man nun damit konfrontiert wurde, die Kinder in einer fremden Umgebung aufziehen zu müssen. Man konnte nicht mehr wie in der Türkei darauf vertrauen, dass die Kinder die eigenen Normen und Werte in der weiteren Umgebung aufgreifen würden. »Gurbet« stand hier für die Angst, die Kinder zu verlieren. Kurz: Die Migrationssituation erzwang eine Besinnung auf die eigenen Normen und Werte. Die von den Gemeinden angebotenen Korankurse spielten hier eine große Rolle. Sie waren, wie die folgende, aus dem Jahre 1977 stammende Äußerung eines Migranten zeigt, weit mehr als eine Unterweisung in Glaubensgründen: »Zwischen Kindern [die zum Korankurs gehen] und anderen gibt es einen sehr großen Unterschied, was die Erziehung, was die Achtung gegenüber dem Vater betrifft, was die Achtung dir [als Gast] gegenüber betrifft. Wenn ein Gast das Haus betritt, wird das Kind ihn respektieren. Aber ein anderes Kind

7) $\mathrm{Zu} »$ gurbet« als Schlüsselbegriff bei Migranten der ersten Generation siehe Greve 2002. 
wird unnötig losquatschen, es wird einem auf die Nerven gehen, es wird einen zornig machen... Also es handelt sich um unsere Sitten und Gebräuche. Die lernt ein Kind im Korankurs. Es lernt sie nicht in der Schule.«(zitiert bei Schiffauer 1993:243)

So weit die religiöse Bedürfnislage, die aus der Migrationssituation resultierte: Sie gab der Religiosität der ersten Generation eine dezidiert defensive Einfärbung. Sie zentrierte sich um Behauptung und Wahrung der eigenen Werte und Lebensentwürfe in einer fremden Umwelt.

Dieses Bedürfnis ließ Migranten überall in der Bundesrepublik die Initiative ergreifen, Moscheen zu gründen. Diese Initiativen wurden in der Regel von unten getragen ${ }^{8}$, von Migranten, die institutionell nicht weiter gebunden waren. Die Initiatoren standen schnell vor dem Problem, dass die Gründung einer Organisation in einer fremden Umgebung Knowhow erfordert. Man musste einen Verein gründen, sich eine Satzung geben, braucht juristische Beratung etc. Dieses praktische Handlungsbedürfnis wurde nun von Organisationen aufgegriffen, die ihren Ursprung in der Türkei hatten und in Deutschland aktiv wurden. Wichtig waren die Süleymanc1, die Nurcu, die Milli Görüș und die Idealistenvereine beziehungsweise »Grauen Wölfe«:

- Die in der Türkei verbotenen Süleymancı und die Nurcu entstammen der islamischen Bruderschaftsreligiosität. Sie waren schon während der ersten Jahre nach der kemalistischen Revolution gegründet worden, um dem, was sie als Verarmung des Islam empfanden, eine islamische Erziehung entgegenzusetzen, und operierten seitdem im Untergrund.

- Die Milli Görüş war in den sechziger Jahren mit dem Ziel gegründet worden, die Islamisierung der Türkei durchzusetzen. Aus ihr sind nacheinander eine Reihe islamisch-konservativer Parteien hervorgegangen (Nationale Ordnungspartei, Nationale Heilspartei, Wohlfahrtspartei, Tugendpartei und schließlich Wohlergehenspartei) Die Abfolge der Namen reflektiert die prekäre rechtliche Stellung dieser Partei in der Türkei: Sie wurde immer wieder verboten und unter einem neuen Namen wiedergegründet.

- Zu diesen drei islamistischen Organisationen gesellten sich die Idealistenvereine (bzw. Graue Wölfe), der europäischen Zweig der rechtsnationalistischen Nationalen Bewegungspartei, die für eine Synthese von Turkismus und Islam eintritt.

- Bemerkenswerterweise wurde das Staatliche Amt für Glaubensangelegenheiten, der Sachwalter des kemalistischen Islam, in den ersten Jahren nicht von sich aus aktiv (wenn auch Gläubige sich bei der Moscheegründung an das Amt wenden konnten, um Hilfestellung zu erhalten). Der türkische Staat überließ also den Gemeinden des politischen Islam weitgehend das Feld. Dies änderte sich erst Anfang der achtziger Jahre, als die Türkei von einer Politik der weitgehenden Ignorierung der Migranten zu einer konservativen Kulturpolitik überging, deren Ziel es war, die Bindung der Auslandstürken an den türkischen Staat zu erhöhen ${ }^{9}$ : Die DITIBB wurde als europäische Niederlassung des Amtes gegründet. Sie steht für einen Islam, der die Rolle von Religion strikt auf den privaten Raum beschränkt wissen will.

- Als letzte Organisation kam schließlich die radikal-islamistische Gemeinde des Cemaleddin Kaplan, der spätere Kalifatsstaat, hinzu, der sich 1983 von der Milli Görüș abspaltete. Er strebte für die Türkei eine islamische Revolution nach iranischem Vorbild an.

8) Nur ausnahmsweise ging bei der ersten Welle der Moscheegründungen die Initiative von transnationalen Organisationen aus: Freilich erfolgte die Bindung an eine Organisation sehr schnell, wenn der Anstoß von Personen, die sich der Milli Görüș oder den Süleymancı verbunden sind, ausging. Ich habe dies am Beispiel von Augsburg dargestellt (Schiffauer 2000:17-26).

9) Es muss darauf hingewiesen werden, dass in den neunziger Jahren bis auf den Kalifatsstaat alle Gemeinden der Einführung der Scharia in der Türkei abschworen. 
Mitte der achtziger Jahre hatte sich das Feld sortiert. Fast alle Moscheen hatten sich der einen oder anderen Organisation zugeordnet. Die Repräsentation des Islam der Arbeitsmigranten durch diese Organisationen hatte eine weit gehende Konsequenz. Das Bedürfnis nach einer defensiven Religiosität, das Europa den Rücken zukehrte, wurde aufgegriffen und auf eine klare Türkeiorientierung hin zugespitzt. Die Gemeinden unterschieden sich darin, welche Rolle sie für den Islam in der Türkei sahen. Gemeinden, die ein affirmatives Verhältnis zur laizistischen Türkischen Republik hatten, distanzierten sich von Gemeinden, die zumindest in den achtziger Jahren eine islamistische Umgestaltung anstrebten. Letztere unterschieden sich im Einzelnen im Hinblick auf die Strategie, die sie für die Einführung der Scharia ins Auge fassten: Die Nurcu und die Süleymanc1 setzten auf consciousness raising durch Korankurse und Ausbildungswerke; die Milli Görüș auf den parlamentarischen Weg, der Kalifatsstaat wiederum auf eine Revolution.

Gegenüber dieser türkeiorientierten Perspektive trat das Engagement für die Rolle, die der Islam in Deutschland spielen sollte, zurück. Besonders deutlich wird dies an den gescheiterten Versuchen, Religionsunterricht an den deutschen Schulen zu etablieren. Ein derartiger Unterricht wäre ganz im Sinne einer defensiven Religiosität gewesen, weil er zur Wertevermittlung in der Fremde beigetragen hätte. Er hätte sich auch gegenüber der deutschen Gesellschaft durchsetzen lassen, wenn die islamischen Gemeinden sich zusammengefunden hätten und gemeinsam als Ansprechpartner deutschen Institutionen gegenüber aufgetreten wären (Schiffauer 1997). Die Interessengegensätze in Bezug auf die Türkei machten dies jedoch unmöglich. Auch dieser Umstand trug dazu bei, dass das Einwanderungsland nicht als »eigenes « Land wahrgenommen wurde, als ein Raum, den man irgendwie aktiv mitgestalten könnte. Deutschland war und blieb »gurbet«, die als leidvoll wahrgenommene Fremde.

Wenn im Exil auch türkischstämmige Organisationen aktiv wurden, so unterschied sich der Exilislam doch deutlich vom Islam in der Türkei und zwar sowohl, was den gelebten Islam betrifft wie auch was die Stellung der islamischen Organisationen angeht. Wichtig war vor allem die Vielzahl von Gemeinden, die in Europa in der Öffentlichkeit agieren konnten. Das in der Türkei existierende Monopol des Staatlichen Amtes für Glaubensangelegenheiten war in Europa gebrochen, auch wenn nach wie vor die größte Zahl der Muslime ihm treu bleiben. Dabei kam in Europa nicht nur eine ohnehin existierende latente Fraktionierung des Islam zum Ausdruck. Gruppen wie die Süleymancı oder auch die Milli Görüș sind in Europa prozentual stärker vertreten als in der Türkei. Bemerkenswert war auch die Gründung des Kalifatsstaats, die in der Türkei keine Entsprechung hatte. Es handelt sich bei ihm um ein Phänomen, das man im Anklang an Benedict Andersons »long distance nationalism « (Anderson 1998) »long distance religiousness « nennen könnte. In der Sicherheit und der Distanz der Migrationssituation wurden bei einem Teil der Gläubigen kompromisslose Positionen entwickelt, die in der Türkei selbst unplausibel waren. ${ }^{10}$

Ein derartiger Pluralismus zieht von selbst ein bewussteres Verhältnis zur Religion nach sich als es in der Türkei existiert: Man hat die Option zwischen verschiedenen Gemeinden. In Europa entfaltet sich damit die Wettbewerbssituation zwischen religiösen Anbietern, die Peter Berger (1967/1973) als charakteristisch für die moderne Religiosität ansieht. Auf dieses strukturelle Moment werde ich weiter unten zurückkommen. Es wird seine Wirkung voll mit der Veränderung der religiösen Bedürfnislage der zweiten Generation entfalten.

Eine größere institutionelle Aufsplitterung wirkte sich allerdings negativ auf die Debattenkultur aus. In der Türkei, wo die Parteiungen unterirdisch existierten, kam es zu einem lebhaften Austausch von Positionen und zu einer lebendigen Debattenkultur. In Europa, wo die

10) Dieser Pluralismus ist ein strukturelles Kennzeichen von Diaspora-Religionen (Vertovec 2002:13). Er reflektiert die Rekonstituierung von Religion in einer neuen Situation. 
Parteiungen manifest waren, nahm die Exit-Option (Hirschman 1974) zu. Man betonte die Grenzen zueinander, distanzierte sich voneinander und bekämpfte sich gegenseitig. Während also die Auseinandersetzungen zwischen den Positionen in der Türkei oft diskursiv geführt wurden, waren sie in Europa oft nicht-sprachlich. Die Gespaltenheit des europäischen Islam wurde damals in der Türkei mit Verwunderung registriert. ${ }^{11}$

Die Idee eines Exil-Islam wurde von den verschiedenen Gemeinden explizit auf den Begriff gebracht. In den DİTIBB-Moscheen nahm sie die Form einer Heimatrhetorik an. Die Vermittlung islamischer Normen und Werte wurde mit der Sozialisation zum Türken und der Vermittlung von Vaterlandsliebe identifiziert. So wurden in der religiösen Unterweisung Unterrichtseinheiten zu Themen wie »Wir lieben unser Vaterland « oder »Liebe zum Vaterland - Die Pflichten gegenüber dem Vaterland - Auch in der Fremde denken wir an die Heimat« angeboten. In der Milli Görüș wurde zu Beginn der Achtziger die Differenz zwischen Eigenem und Fremdem auf die bezeichnende Opposition von dar al Islam - »Land des Islam« und dar al harb - wörtlich »Territorium des Kriegsgeschehens «12 gebracht. Der dominante Terminus in dieser Opposition ist »Dar al Islam«. Er formulierte in Bezug auf die Türkei das damalige politische Programm der Milli Görüș: Die Türkei ist nach dieser Auffassung »eigentlich« ein islamisches Land. Es ist von den Kemalisten entfremdet worden. Der Kampf um die Islamisierung ist deshalb ein legitimer Kampf: Es geht um die Wiederaneignung des eigenen Raums. Der sekundäre Terminus, also »Land des Feindes«, klingt militanter als er gemeint war: Er drückt aus, dass man sich in Deutschland im Prinzip in einem feindseligen Land befand - einem Land, das einem nicht gehörte und auf das man keinen Anspruch haben und das man deshalb nicht gestalten würde. Diesem Land braucht man sich nicht verpflichtet zu fühlen. Eine dritte Formel, mit der der Exil-Islam auf den Punkt gebracht wurde, war der Begriff des Hedschra, der vor allem in der Kaplan-Gemeinde populär war. Hedschra bezieht sich auf den Akt der Auswanderung der jungen islamischen Gemeinde nach Medina im Jahr 622, ein Akt, der durch die politische Unterdrückung notwendig gemacht worden war. Diese Auswanderung findet zehn Jahre später mit der triumphalen Rückkehr nach Mekka ein Ende. Vor allem während des Ausnahmezustands in der Türkei zwischen 1980 und 1983 war diese Definition populär.

\section{Die Entwicklung des Diaspora-Islam}

Auf dem Hintergrund des Exil-Islam der ersten Generation entfaltet sich der wesentlich komplexere Diaspora-Islam der zweiten Generation. Komplexer deshalb, weil die Fraktionierungen, die aus der türkeispezifischen Perspektive resultieren, beibehalten werden, aber nun durch Positionierungen, die sich aus der Auseinandersetzung mit der Einwanderergesellschaft ergeben, überlagert werden. Für die Entfaltung dieser Positionen ist, dies soll im Folgenden am Beispiel der Muslime in Deutschland ${ }^{13}$ gezeigt werden, der Kampf um Anerkennung entscheidend. ${ }^{14}$

Die zweite und dritte Generation von Muslimen steht vor der Situation, dass sie - anders als die Eltern - Europäer sind: Sie sind in der einen oder anderen europäischen Gesellschaft aufgewachsen, sind durch ihre Institutionen gegangen und haben vielfältige Beziehungen zu

11) Siehe etwa die Bestandsaufname bei Mumcu (1987).

12) Der Terminus wurde in der Regel metaphorisch gebraucht, nämlich für Gebiete, in denen die Herschaft nicht islamisch ist oder in denen die Mehrheit der Bevölkerung sich nicht zum Islam bekennt.

13) Die Frage der Anerkennung der islamischen Einwanderer ist in keinem europäischen Land gelöst. Die konkrete Ausbuchstabierung des Kampfes hängt jedoch von den unterschiedlichen politischen Kulturen ab. Siehe hierzu Schiffauer et al. 2002.

14) Zur zentralen Rolle der Anerkennung siehe vor allem Taylor 1992; Honneth 1992; Düttmann 1997. 
der Gesellschaft hier aufgebaut. Sie sind deutsche, englische, französische Muslime und nicht nur Muslime in Deutschland, England oder Frankreich. Dies ist nun nichts Gegebenes, sondern ein praktisches Verhältnis - eine Aufgabe oder ein Projekt. Sie müssen sich als Muslime in der jeweiligen Gesellschaft verorten und in dieser Verortung ein Selbstverhältnis entwickeln. Diese Aufgabe wird nun allerdings durch zwei Faktoren verkompliziert. Erstens wird sowohl von der Einwanderergesellschaft wie von der ersten Generation das Verhältnis von »europäischer Kultur« und Islam als Verhältnis von Eigenem und Fremdem konstruiert - und damit in ein Verhältnis von Opposition (und nicht von Komplementarität) gesetzt. Der zweite Faktor ist, dass dieses Verhältnis von einer Machtasymmetrie beherrscht wird. Es handelt sich nicht um ein Verhältnis unter Gleichen, sondern die europäische Seite ist ungleich mächtiger. Neuankömmlinge, die einen Platz für ihre Religion erkämpfen wollen, sind immer in einer strukturell benachteiligten Situation gegenüber den Platzinhabern, die die Bedingungen für die Zulassung definieren.

Die Konstruktion des »Muslim-Other « ist immer wieder analysiert worden und braucht deswegen hier nur angedeutet zu werden. Sie macht sich heute insbesondere an zwei Feldern fest, die als zentral für die europäische Wertegemeinschaft gelten. Zum einen existiert der Verdacht einer prinzipiellen Demokratieunfähigkeit auf Grund einer nicht vollzogenen Aufklärung. Die Trennung von Politik und Religion ist, so die Unterstellung, dem Islam im Wesentlichen fremd. Der zweite große Bereich ist der der Gleichheit von Mann und Frau. Die islamische Familie wird als Hort von Autoritarismus, Patriarchalismus, Frauenfeindlichkeit und häuslicher Gewalt gesehen - als der genaue Gegenentwurf zur egalitären und »befreiten « europäischen Familie. Dem Islam als Weltreligion wird zwar prinzipiell ein Wert zugestanden - im Einzelnen fällt es den meisten Europäern schwer, sich vorzustellen, welchen schätzenswerten Beitrag der Islam zu der europäischen Zivilgesellschaft leisten könnte oder was Europäer vom Islam lernen könnten. Dabei paart sich das Misstrauen mit Angst um die eigene Identität: Man fürchtet einen wachsenden Einfluss der Muslime; an Äußerungen, die das belegen, gibt es keinen Mangel. Vielleicht lohnt es sich, eine zu zitieren, weil sie von einem sozialdemokratischen und der Migration insgesamt positiv gegenüberstehenden Politiker stammt.

»Ich glaube, dass die Frage Islam/Islamunterricht zugleich, das spürt man als Politiker, große Ängste und Besorgnisse in unserer Bevölkerung aktiviert. Das spürt man insbesondere, wenn man zu Diskussionen in Stadtquartieren in Berlin unterwegs ist. Da gibt es - das sage ich zugespitzt - die Sorge, dass sich über Islam, über Menschen islamischen Glaubens in Deutschland, die dann auch noch in die Schule kommen, die Art und Weise unserer Kultur, die ja durch und durch abendländisch geprägt ist, gewissermaßen schleichend verändert.« (Klaus Böger in Senatsverwaltung 2000:4)

Diese Denkfiguren bestimmen die Debatte auf den unterschiedlichsten Ebenen: Bei fast jeder Debatte über Identität und Grenzen Europas - etwa im Zusammenhang mit einem EUBeitritt der Türkei - wird der Grundverdacht geäußert, dass die judeo-christliche und die islamische Wertgemeinschaft unvereinbar seien. Der generalisierte Fundamentalismusverdacht hat nach dem 11. September eine bestimmte Zuspitzung erfahren. Besonders entscheidend dürfte jedoch sein, dass der Alterierungsdiskurs auch die schulische Realität zumindest in einigen Ländern Europas nachdrücklich prägt (Schiffauer et al. 2002).

All dies führt zu einem verbreiteten Gefühl bei der zweiten Generation, dass man doppelt diskriminiert ist, nämlich sowohl als Einwanderer als auch als Muslim. Die Europäer würden sich früher oder später mit der Einwanderung von säkularen Türken oder Arabern abfinden, nicht aber mit der Einwanderung von bekennenden Muslimen, meinte ein junger Muslim der zweiten Generation zu mir. ${ }^{15}$ 
Die Abwertung wäre allerdings weniger problematisch, wenn sie nicht mit der Machtdifferenz verbunden wäre, die Neuankömmlinge von Platzinhabern trennt. Es geht um die Forderungen einer Minderheit, die gegen die herrschenden Selbstverständlichkeiten durchgesetzt und einer skeptischen Mehrheit oft gegen ihren Widerstand abgetrotzt werden müssen. Dies wäre an sich nicht so problematisch. Im Unterschied zu vielen anderen Minderheiten werden die Muslime jedoch mit einem bemerkenswerten gesellschaftlichen Schulterschluss konfrontiert. Anders als bei anderen Fragen, die Immigranten betreffen, fehlen den Muslimen die Koalitionspartner auf der Seite der Linken ${ }^{16}$. Im Gegenteil: In Bezug auf den Islam sind die aus einer Mischung von Säkularismus und Feminismus gespeisten Einwände der Linken oft massiver als die von Seiten der Rechten. So haben viele Muslime oft das Gefühl, gegen eine Mauer anzurennen, wenn sie Forderungen erheben - beginnend beim Bau von Moscheen, der gegen den expliziten Widerstand der Anrainer, manchmal auch den der Behörden durchgesetzt werden muss, über das Recht auf islamische Kleidung in der Schule oder am Arbeitsplatz bis hin zu dem Wunsch, die Schamgrenzen etwa beim Schwimm- oder Sportunterricht respektiert zu sehen. In all diesen Bereichen erleben sie, dass ihre Forderungen auf Skepsis, Zurückhaltung und nicht selten auf Widerstand stoßen. Nicht selten ergibt sich die Situation, trotz eindeutiger Rechtslage auch von Behörden hingehalten zu werden oder das, was man als Recht erachtet, nur über langwierige Gerichtsverfahren durchsetzen zu können. Die Konfrontation mit Macht bedeutet bei solchen Auseinandersetzungen häufig gerade auch die Begegnung mit Definitionsmacht. Nicht selten muss man es sich in solchen Auseinandersetzungen als Muslim gefallen lassen, dass die eigenen Anliegen - aber auch man selbst - von Nicht-Muslimen danach klassifiziert und beurteilt werden, ob sie dem »eigentlichen humanen Islam« entsprechen oder »fundamentalistisch pervertiert « sind.

Unter den geschilderten Bedingungen, so meine These, spitzt sich die Suche nach Anerkennung fast notwendigerweise zu einem agonalen, konflikthaften Kampf um Anerkennung zu. Das Verhältnis von Macht und Gegenmacht wird in solchen Situationen zentral. Es kommt zu einer Verhärtung, die eine zwanglose Ausprägung von Identität nicht möglich macht. Bevor wir uns dem Material zuwenden, muss dieses Problem kurz entfaltet werden. ${ }^{17}$

Die Suche nach Anerkennung bezieht sich auf ein prekäres Verhältnis von Gleichheit und Differenz. Man will als Gleicher anerkannt werden - weil Ungleichheit meist Ausgrenzung und Diskriminierung bedeutet. Man will aber auch als besonders und einmalig wahrgenom-

15) Das Bild wäre allerdings unvollständig, wenn man nicht auch sehen würde, dass ebenso verzerrende Bilder des Westens von der ersten Einwanderergeneration gezeichnet werden. Die »europäische Kultur« wird als Inversion zur eigenen islamisch-türkischen Kultur konstruiert - als Hort der sexuellen Libertinage, der Freizügigkeit in Bezug auf Alkohol und Drogen und des Verfalls der familialen Bindungen.

16) Es gibt natürlich Ausnahmen, z.B. in der Person der Bundesausländerbauftragten Marie-Luise Beck (Bündnis 90/DIE GRÜNEN). Die Geschichte von islamischen Gemeinden und der Linken ist vielschichtig und kompliziert. In der Türkei standen sich in den siebziger Jahren die Linke und islamische und islamistische Gemeinden als Gegner gegenüber. Dabei hielten sich die islamischen Gruppen zwar weitgehend aus den blutigen innenpolitischen Auseinandersetzungen zwischen Rechten und Linken heraus. Dennoch war die Kluft in Bezug auf Lebensstile und Lebensentwürfe zwischen islamischen Gemeinden und der Linken größer als zwischen letzterer und den oft säkularen rechten Gruppen. Mit der Migration nach Deutschland hatten die türkischen Linken in Gewerkschaften und den Parteien (vor allem der SPD) maßgebenden Einfluss auf die Ausprägung der Positionen dieser Gruppen gegenüber den islamischen Gemeinden. Die Muslime wiederum zogen sich in die Gemeinden zurück, die sie als Inseln im Meer von Ungläubigen wahrnahmen. Die zweite Generation hat heute die Konsequenz dieser Auseinandersetzungen zu tragen.

17) Es ist im Rahmen dieses Textes nicht möglich, auf alle Fragen einzugehen, die in der umfangreichen sozialphilosophischen Literatur zu dem Problem von Macht und Anerkennung diskutiert wurden. Besonders wichtig sind hier Goffman 1963/1980; Bhabha 1993; Butler 1997; Fanon 1981. 
men oder zumindest in seiner Besonderheit respektiert werden. Die amerikanische Redewendung »to make a difference «, mit der Anerkennung ausgedrückt wird, bezeichnet dies sehr genau. Es ist deutlich, dass in diesem doppelten Anerkennungsbegehren eine Spannung angelegt wird. Es scheint mit dem Problem der Quadratur des Zirkels vergleichbar zu sein. Kaum wird man als different und besonders gesehen, stellt sich das Problem der Gleichheit; kaum ist man als gleich behandelt, stellt sich die Frage nach dem Recht auf Differenz, erwacht das Unbehagen darüber, dass Unvergleichbares über den gleichen Kamm geschoren wird. Nun wird dies gelegentlich zu einer Paradoxie stilisiert (etwa bei Düttmann 1997). Ich würde nicht so weit gehen. Das Verhältnis von Gleichheit und Differenz ist nämlich dann unproblematisch, wenn die Besonderheit Anerkennung findet oder zumindest auf eine gewisse wohlwollende Offenheit und Neugier stößt. Dies ist dann der Fall, wenn, wie Charles Taylor (1993:66) es formuliert, man sich im Kulturkontakt von der Annahme leiten lässt, dass die jeweils andere Kultur einen Wert besitzt, was nicht heißt, dass man dann schließlich zu dem gleichen Urteil kommen muss. Der Zen-Buddhismus beispielsweise erfreut sich in Europa einer grundsätzlichen Anerkennung, und bestimmte Praktiken, die auf seinem Hintergrund erwachsen, können mit einer neugierigen, grundsätzlich positiv gestimmten Haltung eines weiten Teils der Öffentlichkeit rechnen ${ }^{18}$. Jemand, der sich dieser Religionsgruppe zuordnet, kann seine Besonderheit ausspielen, ohne dass die Frage der Gleichheit aktuell wird. Wenn dies jedoch nicht so gesehen wird - wie im Fall des orthodoxen Islam -, dann treten die Anerkennung von Differenz und die Anerkennung von Gleichheit in ein fast unauflösbares Dilemma. Dann nämlich geht das Betonen von Besonderheit auf Kosten der Gleichheit - und umgekehrt. Die Betonung der Besonderheit wird dann nicht als besonderer oder möglicher Beitrag zur »Wertegemeinschaft«, sondern als Verletzung ihrer Prinzipien gesehen. Der Schritt zur Ausgrenzung ist dann leicht. Eine Vierzehnjährige, die sich für das Tragen einer streng islamischen Kleidung entscheidet, gehört dann irgendwie nicht mehr zu der deutschen Klassengemeinschaft. Sie wird in einem elementaren Sinn nicht mehr als gleich empfunden; sie ist »anders«, aber nicht »besonders«. Umgekehrt stellt sich bei denjenigen, die versuchen, als Gleiche anerkannt zu werden, sehr schnell und leicht das Gefühl ein, dass dies nur möglich ist, wenn man seine Besonderheit und damit sich selbst verleugnet. Auf dieser Folie kommt man dann leicht zu der Position, dass die Suche nach Anerkennung per se schon ein Zwangsverhältnis impliziert.

Auf dieser Folie werden die Identitätsdilemmata verständlich, in die sich die zweite Generation in der Auseinandersetzung mit der europäischen Gesellschaft verstrickt. Um es zugespitzt zu formulieren: Die europäischen Gesellschaften machen es fast unmöglich, sich ohne Windungen, Verdrehungen oder Selbstverleugnungen als europäischer Muslim zu definieren oder, wie man es abstrakter formulieren könnte, eine selbstverständliche islamische Identität in Europa auszubilden. Ich möchte dies zeigen, indem ich idealtypisch die Identitätsoptionen durchmustere, die von der zweiten Generation in der Auseinandersetzung mit der europäischen Gesellschaft entwickelt werden. In guter Weberscher Tradition werde ich die Positionen sich gegenseitig kommentieren und kritisieren lassen. Es wird dabei deutlich werden, dass jede der Positionen sich als Antwort auf die Schwierigkeiten versteht, die sich aus den anderen Positionen ergeben - nur um selbst wieder in Sackgassen zu führen.

\section{Option 1: Der Kampf um Gleichheit}

Die vielleicht naheliegendste Forderung für eine religiöse Minderheit ist der Kampf um Gleichheit und Gleichberechtigung. Der islamische Lebensentwurf soll nicht weniger anerkannt werden als der christliche oder der jüdische. Man muss sich als Muslim - individuell

18) Siehe zur unterschiedlichen Wahrnehmung von Islam und Buddhismus auch Klinkhammer 2000:253. Weitere Verweise dort. 
oder kollektiv - an den gesellschaftlichen Debatten der Bürgergesellschaft über Abtreibung, Scheidungsrecht etc. genauso beteiligen können wie andere religiöse Gruppen, und den islamischen Stellungnahmen muss das gleiche Gewicht zugemessen werden wie den jüdischen und christlichen. Es geht dabei im Wesentlichen um Individualrechte in einer offenen Bürgergesellschaft. Diese Individualrechte umfassen auch eine »von den Bürgern selbst gesteuerte Gleichbehandlung ihrer identitätssichernden Lebenskontexte«, wie es Jürgen Habermas formulierte (1992:158). Tatsächlich hat man den Eindruck, dass die Vertreter dieser Position, wenn man ihre Äußerungen auf ein implizites Gesellschaftsideal hin abtastet, den Habermas 'schen Traum weitgehend teilen. Diese Position stellt den Kampf gegen Diskriminierung, also gegen jede Form von Ungleichbehandlung, in den Vordergrund.

Diese Position ist für Muslime besonders plausibel, die den Islam als Privatsache, als Angelegenheit zwischen Individuum und Gott betrachten. Diese Position ist notwendigerweise werteplural, insofern sie es ablehnt, das gesellschaftliche Leben methodisch vom Standpunkt der Religion her durchzugestalten. Dies geht Hand in Hand mit einer Subjektivierung und Privatisierung des islamischen Kults. Eine systematische Studie zu einem derartigen Islamverständnis steht noch aus. ${ }^{19}$ Hinweise, die freilich nicht mehr als ein Schlaglicht auf das Phänomen werfen, lassen sich einer Fallstudie entnehmen, die in jüngerer Zeit von Aylin Gençel angefertigt wurde (2003). Die von ihr beschriebenen Mitglieder einer Berliner türkischstämmigen Familie bekennen sich zu einem »bewussten« Islam. Sie meinen damit einen Islam, dem man sich aus eigener Entscheidung zuwendet, und vor allem einen Islam, den man sich eigenständig aneignet. Der »bewusste Islam« setzt sich von einem traditionalen (sie sagen: »dörflichen«) Islam ab, den man ohne viel zu reflektieren und zu prüfen von seinen Eltern übernimmt. Die Mitglieder der Familie folgern aus der individuellen Hinwendung zu Gott, dass es im Islam keinen Zwang gibt. »Dann gibt es das auch nicht, dass einer den anderen zu etwas zwingt. Warum bete ich denn den namaz? Für meinen eigenen Seelenfrieden. Warum faste ich? Ich faste nicht für dich, meine Mutter, die Kinder oder meinen Mann. Sondern ich faste für mich.« (nach Gençel 2003:44) In der familialen Praxis sieht dies so aus, dass Frauen mit Kopftuch und Frauen ohne Kopftuch zusammenleben; dass die älteren regelmäßig beten, während die jüngeren sich damit noch Zeit lassen. Die Kinder werden zum Korankurs geschickt. Aber es wird auch toleriert, wenn die Tochter einen Nicht-Muslim heiratet - und die Familie setzt sich mit einem gewissen Stolz auf ihre Toleranz von Verwandten ab, die diese Entscheidung verurteilen. Die Mitglieder der beschriebenen Familie sind ebenfalls skeptisch gegen eine zu starke Stellung der islamischen Gemeinden. Sie sehen in ihnen Institutionen, die sich prinzipiell zwischen Individuum und Gesellschaft stellen und oft dirigistische Ansprüche stellen. Man wirft den Gemeindeangehörigen »Autoritätsgläubigkeit « vor: Die Gläubigen würden oft blind den Hodschas folgen, ohne sich selbst ein Bild zu machen. Der Dogmatismus, der, so die Vertreter dieser Position, die Gemeinden charakterisiere, ist mit ihrer eigenen Form der individuellen Aneignung nicht vereinbar.

Das hier formulierte Ideal der individuellen Aneignung der Tradition ist weit verbreitet, auch wenn viele einräumen würden, dass sie selbst - leider - nicht die Zeit dazu fänden, es auch in der Praxis umzusetzen. Dies geht einher mit einer gegenseitigen Respektierung unterschiedlicher Lesarten. All dies gilt als Voraussetzung dafür, den Islam so weiterzuentwickeln, dass er in die Moderne passt - was auch die Voraussetzung dafür ist, den wichtigen Beitrag des Islam zur Moderne zur Geltung zu bringen. »Der Westen hinkt hinter dem Koran her; aber wir hinken hinter dem Westen her« ist eine gerne bemühte Redewendung, die dies zum Ausdruck bringt.

19) Dies ist kein Zufall. Das Phänomen dieses Mehrheitsislams (musluman de culture) lässt sich naturgemäß schwieriger beschreiben, als der kommunalistische oder revolutionäre Islam, weil er keine eigenen Organisationsformen und Dogmen hervorbringt. 
Diese Vision eines individuierten Islam ist wahlverwandt zu einem »liberalen« Islam, d.h. einem Islam, der sich selbstbewusst zu Wertepluralität, Stimmenvielfalt und individuell besonderen Glaubenswegen bekennt. Damit eine derartige Position zur Entfaltung gelangt, wäre es allerdings notwendig, dass das oben entfaltete Problem von Macht und Diskriminierung gelöst wird. Nur dann wird eine derartige Position nicht mit Selbstaufgabe, Assimilation und Schwäche assoziiert werden. Die europäischen Gesellschaften müssten den Islam als ernstzunehmende Stimme zulassen. Dies würde u.a. die Bereitschaft erfordern, der Habermas 'schen Forderung zu entsprechen, die universalistischen Gehalte ihrer Verfassungen von den lokalen und partikularistischen Traditionen zu trennen - einmal vorausgesetzt, dass dies überhaupt möglich ist (Habermas 1992:166ff.). Im Prinzip ist die englische Gesellschaft mit ihren Versuchen, Britishness neu zu definieren, in Europa bei diesem Versuch am weitesten gegangen. In den kontinentalen Gesellschaften ist dies noch kaum sichtbar.

Die Konfrontation mit islamophoben Zuschreibungen, mit Ausgrenzungs- und Ohnmachtserfahrungen stellt die Vertreter eines individuierten Islam vor das Problem, für ihre Positionen kämpfen zu müssen. Wie wir dies bei der Darstellung der nächsten Position sehen werden, hieße dies aber, einige ihrer Grundprinzipien opfern zu müssen. Wenn sie nicht dazu bereit sind, führt dies zu einem fast resignativen Rückzug. Man lebt dann, wie die von Aylin Gençel beschriebene Familie, seinen Islam im Privatraum. Dort wird dann eine fast perfekte türkische Welt (mit dezidiert urbanem Charakter) gelebt. Sie leben in Berlin und halten zu der deutschen Gesellschaft technische Beziehungen aufrecht. Anpassungsforderungen wird eine entschiedene Abfuhr erteilt:

»Die größe Frage ist die Anpassungsfrage an die Deutschen. Nun ist der Ministerpräsident Mesut Yilmaz gekommen und hat folgendes verkündet: >Die Türken müssen sich an die Deutschen anpassen. Also in welcher Hinsicht sollen wir uns anpassen? OK - mit der Sprache, das akzeptiere ich. Aber in Bezug auf Arbeit oder Leben, was weiß ich - in welchem Grade sollte ich mich anpassen? Ich weiß nicht - aber wenn ich das höre, empfinde ich Widerwillen. Also ich lebe hier, ich muss Deutsch sprechen können..aber abgesehen davon, wieso sollte ich mich einem Deutschen anpassen? Ich glaube nicht, dass meine Kinder, ich, mein Gatte, uns an die Deutschen anpassen müssen. Ich sehe die Notwendigkeit nicht. Ich arbeite in diesem Land, zahle meine Steuern, ziehe meine Kinder auf, schicke sie zur Schule, einer von ihnen arbeitet schon, aber ich habe nicht wie die Deutschen gelebt ..ich lebe wie ein Türke und habe auch noch keine Reaktion von deutscher Seite darauf bekommen.« (unpubliziertes Interview von Gençel 2003) Die Anpassungsleistung, die für Vertreter des individuierten Islam im Grunde unproblematischer zu erbringen wäre als für Vertreter des kommunalistischen Islam, weil religiöse Normen niedriger gehängt oder weniger streng beachtet werden, wird dann zum Problem, wenn sie in der Konfrontation mit einem Machtdiskurs in den Ruch symbolischer Unterwerfung kommt. ${ }^{20}$

\section{Option 2: Der Kampf für das Recht auf Differenz}

Die Vertreter der zweiten Position, die man mit »kommunalistisch engagiert« bezeichnen könnte, setzen an dieser Stelle an. Sie halten die individuierte Position für aussichtslos. Ist es wirklich realistisch zu meinen, die Differenz im Privatraum leben und behaupten zu können? Hat man dann nicht von vorne herein verloren? Man verbirgt den Islam, die Religion, wie ein Stigma, dessen man sich schämen müsste. Es ist klar, dass die deutsche Gesellschaft mit

20) $\mathrm{Zu}$ dieser Reaktion tendieren auch durchaus säkulare Muslime. Unvergessen geblieben ist mir der Wutausbruch eines jungen Berliner Türken: »Wer sind wir denn überhaupt...Und wenn die noch so weiter gackern, dann lass ich meine Frau auch Schleier anziehen, obwohl sie Hosen anhat.« (Schiffauer 2002:21) 
den individuierten Muslimen gut leben könnte. Sie bräuchte sich nämlich dann mit dem Islam nicht auseinander zu setzen und würde sich deshalb auch nicht bewegen. In Bezug auf Anerkennung - etwa der Tatsache, dass man für sein Recht eintritt, ein Kopftuch zu tragen kommt man in dieser Hinsicht nicht weiter. Ein Mädchen mit Kopftuch wird auch weiterhin einfach isoliert sein. Deshalb ist es notwendig, dem Islam in der Öffentlichkeit einen Platz zu erkämpfen. Es müssen islamische Räume geschaffen werden. Der Islam muss ein akzeptierter way of life in der europäischen Gesellschaft werden. Eine islamische Kleidung muss die gleiche Selbstverständlichkeit bekommen wie ein Kette mit dem Kruzifix. Ein islamisches Mädchen muss selbstbewusst und stolz ein Kopftuch tragen können. Nur dann wird die Besonderheit als Besonderheit wahrgenommen und nicht mehr als Andersheit gesehen, als eine Differenz, die man ausgrenzen muss. Kurz: Während die Vertreter der ersten Position von der Forderung der Gleichheit ausgehen, stellen die kollektivistisch engagierten Muslime das Recht auf Differenz an erste Stelle und leiten daraus die Forderung nach Gleichheit ab. Wir sind anders und wir haben ein Recht anders zu sein und wir können von der Mehrheitsgesellschaft erwarten, dass sie dieser Andersheit Respekt zollt. Die Auseinandersetzungen beziehen sich hier auf kollektive Rechte der Religionsgemeinschaft - etwa auf das Recht, islamische Kleidung in Schule, Universität und am Arbeitsplatz zu tragen, auf das Recht auf ein Passbild mit Schleier oder Ähnliches.

Nur gemeinsam hat man eine Chance, den Kampf um die Rechte auch zu gewinnen. Als Einzelner ist man dagegen verloren. Aus dieser Einsicht heraus tendieren die Vertreter dieser Position von vorne herein dazu, Solidarität stärker zu betonen als die Vertreter der ersten Position. Wie auch in anderen Gruppen erscheint community building als Möglichkeit von Widerstandspolitik. Was Paul Gilroy für »black Britain« festhält, bezieht sich auch auf die islamischen Gemeinden: »Community, therefore, signifies not just a distinctive political ideology but a particular set of values and norms in everyday life: mutuality, cooperation, identification and symbiosis. For black Britain, all these are centrally defined by the need to escape and transform the forms of subordination which bring >races< into being « (Gilroy 1993:414). Eine nachdrückliche Betonung der Bedeutung des Gemeindelebens folgt daraus. Ein lebendiger Islam ohne ein lebendiges Gemeindeleben erscheint den Kommunalisten undenkbar - sie sehen in der liberal-individualistischen Position einen blässlichen Abklatsch eines geistbeseelten Șuurlu Islam.

Wie Anthony P. Cohen (1985) gezeigt hat, verlangt Gemeindebildung die Konstruktion von Symbolen, auf die man sich einigt und mit denen man sich abgrenzt. Beim kommunalistischen Islam ist die Körpersymbolik zentral. Über die Kleidung, insbesondere der von Frauen, wird ein starkes Symbol der Differenz zur Mehrheitsgesellschaft, aber auch zum liberalen Islam geschaffen. Das Abzeichen ist besonders der »türban«, die besondere Form der islamischen Verhüllung, die in der Türkei nach 1980 aufkam und die sich deutlich vom traditionellen Kopftuch unterscheidet. Dieses Symbol steht allgemein für das Bekenntnis zu einem islamischen Familialismus. Was sich allerdings von außen (und auch in der Selbstwahrnehmung) als homogen darstellt, entpuppt sich bei näherer Betrachtung als sehr heterogen. Wie bei allen anderen Gemeinden stellt die Kleiderordnung eine Gemeinsamkeit der Form her, weit weniger aber der Inhalte. So kann man zeigen, dass sich hinter der Hinwendung zum »türban« sehr verschiedene Motive verbergen (Jouli 2003), die sich manchmal kombinieren, manchmal aber auch auseinander treten. Der »türban« kann für eine Kritik der westlichen Sexualmoral, für eine asketische Körpertechnik oder auch für die empfundene Notwendigkeit stehen, sich sichtbar zum Islam zu bekennen. Besonders interessant, und für unsere Zwecke wichtig, ist es, wenn das Tragen des türban zu einer Voraussetzung für argumentative Rebellion wird. Dies ist bei engagierten feministischen Muslima der Fall. Ich erlebte etwa bei einer Milli Görüș-Veranstaltung, wie mehrere Referentinnen sehr deutlich und massiv gegen Gewalt in der Ehe, gegen arrangierte Heiraten, für das Engagement von Frauen in der 
Öffentlichkeit Stellung bezogen und - um letzteres zu ermöglichen - für die Beteiligung der Männer an der Hausarbeit eintraten. Es war sehr deutlich, dass diese Argumentation vielen der anwesenden Männer zu weit ging. Die symbolische Eindeutigkeit, die die Frauen mit ihrer Kleidung herstellten, zwang die Männer jedoch dazu, sich mit ihren Forderungen auseinander zu setzen. Man hat den grundsätzlichen Punkt der Loyalität geklärt - und kann nun umso nachdrücklicher zur Sache kommen.

Eine kommunalistische Position weist eine gewisse Wahlverwandtschaft mit Orthodoxie und zwar im genauen Sinn von Recht-Gläubigkeit auf. Die Einbindung in die Gemeinde geht mit der Bejahung der gemeinsam verbindenden Gesetzlichkeit einher. Dies ist allerdings alles andere als statisch: Gerade in der Diaspora entfaltet sich hier ein Bemühen darum, wie man rechtlich einwandfreie Lösungen für neue Herausforderungen finden kann. Dem Kampf um das Recht auf Differenz in der Gesellschaft korrespondiert eine theologische Suche nach der Begründung dieses Rechts im religiösen Gesetz und der gleichzeitigen Bejahung der Notwendigkeit, in Bezug auf die Umsetzung die neue Situation zu berücksichtigen, die sich aus dem Leben in Europa ergibt. ${ }^{21}$

Die Betonung von Gemeindebindung geht nicht selten mit einer starken Betonung von Gruppensolidarität einher. Dies steigert sich in Situationen, die von Machtungleichgewicht charakterisiert sind, leicht zu einem Loyalitätszwang. Wer um kollektive Rechte kämpft, tendiert dazu, in innerer Geschlossenheit ein wichtiges Moment zum Aufbau von Gegenmacht zu sehen. Dem fällt in der Regel innere Kritik, insbesondere die an der Führung der Gemeinde, zum Opfer. Eine hohe soziale Kontrolle geht oft mit der Sanktionierung von Aussteigern und Außenseitern einher. Widerspruch wird oft aus Angst vor Ausgrenzung nicht öffentlich formuliert, sondern allenfalls hinter vorgehobener Hand. Dabei wäre es ein Missverständnis, dies auf eine Kontrolle von oben nach unten zu reduzieren. Die Kontrolle wird durchaus auch von unten und aus freien Stücken ausgeübt, nämlich von Gemeindeangehörigen, die in einer intakten Führung den Garant für den Zusammenhalt der Gemeinde sehen. Ein Beispiel für diese Argumentation lieferte die Vertreterin der Muslimischen Jugend, Riem Hawi, auf einer Veranstaltung im Dezember 2003 im Tempodrom. Laut der Journalistin Claudia Dantschke führte sie aus, dass die keine Probleme gehabt habe, solange sie die einzige Muslima an einer Schule gewesen sei. »Als andere Muslime hinzukamen, habe sie immer begründen müssen, warum andere am Sportunterricht teilnehmen würden, sie aber nicht. >Wir müssen enger zusammen arbeiten und konsequenter in unseren Ausführungen sein.« Wie Claudia Dantschke (2004) richtig, wenn auch in völliger Unsensibilität für die eine solche Position begründenden Zwänge, anmerkt, wird darin »ein wenig von dem Gruppenzwang sichtbar,der an manchen Schulen dazu führt, dass muslimische Kinder keine Wurstbrote mehr essen wollen...«

In solchen Situationen wird, um es mit Bourdieu zu formulieren, einer der beiden »genuin politischen Modi«, nämlich der der eigenständigen Meinungsbildung, zugunsten des anderen, der Übertragung der Vollmacht, zurückgenommen, d.h. zugunsten der »Wahl von Wortführern und Bevollmächtigten im Sinn der Entscheidung für bestimmte Ideen, Überzeugungen, Entwürfe, Programme, Pläne, die, da inkarniert in Persönlichkeiten, in ihrer Wirklichkeit und Glaubwürdigkeit eben auch von der Wirklichkeit und Glaubwürdigkeit dieser >Persönlichkeiten< abhängen« (Bourdieu 1982:665).

Die Tendenz zur »Zurückstellung des Selbst« (Bourdieu) scheint mit der Einsicht zusammenzuhängen, dass das Bekenntnis zur Individualität - zur eigenständigen Meinungsbildung

21) Diese Suche findet u.a. im European Council for Fatwa and Research statt, wo ein Islam für Minderheiten definiert wird. Die Islamische Gemeinschaft Milli Görüș ist Mitglied in diesem Gremium (Caeiro 2003). 
- unter bestimmten Umständen die Sache schwächen kann, weil sie der Isolation, Überwachung und Vereinzelung des individuellen Subjekts Vorschub leistet.

Allerdings wird hier die Problematik der Position einer kollektiven Selbstbehauptung deutlich. Sie ist angetreten, um angesichts von Ohnmacht und Diskriminierung eine Gegenmacht aufzubauen. Gleichwohl gibt es eine problematische Tendenz, die Machtstrukturen nach innen zu verdoppeln - und zwar umso ausgeprägter, je größer der Druck der Gesellschaft ist, auf den man antwortet. Eben dies lässt diese Gemeinden leicht zu Orten eines inneren Autoritarismus werden. Unter den Bedingungen der Machtlosigkeit und Diskriminierung schlägt Solidarität leicht in Loyalitätserwartungen um.

Gerade diese »Autoritätshörigkeit« des kommunalistischen Islam erscheint Vertretern eines liberalen Islam häufig problematisch. Andererseits würde, so die Vertreter des orthodoxen Islam, die Position der Liberalen zur Selbstaufgabe führen.

\section{Option 3 : Die Ablehnung des Kampfes um Anerkennung}

Die dritte Position, die man unter den Muslimen der zweiten Generation findet, ließe sich als antihegemoniale charakterisieren. Sie hält den Vertretern eines individuierten Islam und den des kommunalistisch-orthodoxen Islams vor, dass die Suche nach Anerkennung, sei es in Form der Anerkennung von Gleichheit oder von Differenz, immer in die Sackgasse führt. Diese Position ist extrem sensibel gegenüber dem Problem von Macht und Identität, so wie sie am deutlichsten von subjekttheoretischen Denkern entfaltet wurde (Butler 1997/2001; Foucault 1981) ${ }^{22}$.

Sobald man überhaupt beginnt, in der feindlich gestimmten Gesellschaft um Anerkennung zu werben - ob nun um Anerkennung als Gleicher oder als Differenter -, habe man sich schon aufgegeben. Man überlässt nämlich dann dem anderen, der nicht der Gemeinde angehört, die Definitionsmacht darüber, wer als guter und wer als schlechter Muslim zu gelten hat. Dies ist nicht nur kränkend, sondern es ist auch eine Spirale, an deren Ende die Selbstverleugnung steht, weil man sich am Ende den Werturteilen der anderen Religion unterworfen hat. Was aber - so fragen die Vertreter dieser Position - legitimiert die Mehrheitsgesellschaft denn überhaupt dazu, sich als Richter über den Islam aufzuspielen? Ganz sicher nicht die moralische Überlegenheit. Tatsächlich war es einer der zentralen Inhalte der Zeitschrift D.I.A. (»Die islamische Alternative «), die als Organ der mittlerweile verbotenen Hizb-ut Tahrir ein Forum für die revolutionäre Variante diese Position darstellte, die moralische Selbstgefälligkeit des Westens anzugreifen. Welche Gesellschaft hat Faschismus und Kolonialismus hervorgebracht, welche hat den Massenmord an den Juden verübt? Die Gewalt, die den Westen in seiner gesamten Geschichte charakterisiert, richtet sich heute, so die Vertreter dieser Position, primär gegen den Islam. Israel, Tschetschenien, Afghanistan und der Irak werden immer wieder als Beispiele einer imperialistischen Politik aufgeführt. Das Ziel dieser Politik sei es, die einzige Stimme, die sich dem global geltenden hegemonialen Diskurs entgegenstelle, zu zerschlagen. Wer, wie die Vertreter des individuierten oder des kommunalistischen Islam, versuche, sich mit der Hegemonialmacht zu arrangieren (oder sich in ihrem Schatten einzurichten), werde letztendlich das Potenzial des Islam verspielen, eine ra-

22) Hierzu siehe insbesondere Butlers Diskussion von Althussers Szene der Interpellation. Die Suche nach Anerkennung bedeutet immer auch, sich affirmativ zu dem Prozess des asujetissements zu verhalten. In unserem Fall: Die Muslime in Europa werden als Subjekte durch Interpellation der Staatsmacht konstituiert. Wer diese Interpellation als an sich gerichtet annimmt, akzeptiert damit auch »die durch diese Stimme bewirkte Unterordnung und Normalisierung (Butler 2001:11). Die antihegemoniale Position besteht in dem verzweifelten, weil zum Scheitern verurteilten, Versuch, sich dieser Interpellation zu entziehen. 
dikale Alternative zu sein. Dies ist auch theologisch nicht zu rechtfertigen. Man muss an der absoluten Unverhandelbarkeit islamischer Positionen festhalten.

Die antihegemoniale Position ist mit einer Wendung zur Ultraorthodoxie wahlverwandt. Sie betont Reinheit und Authentizität und grenzt sich von weniger puristischen Positionen ab. Die Grenze, die gegenüber der Mehrheitsgesellschaft gezogen werden, werden auch gegenüber den anderen Muslimen gezogen, denen vorgeworfen werde, sich auf das System einzulassen und damit den Islam zu verraten. Die Abgrenzung nach außen produziert einen Dogmatismus nach innen und eine Sanktionierung aller möglichen Abweichungen von der reinen und wahren Lehre. Diese Position existiert in einer weltzugewandten und einer weltablehnenden Variante. Die weltzugewandte Position betont, dass man den wahren Islam nur in einem wahrhaft islamischen Staat leben kann - und leitet daraus die Notwendigkeit zur Revolution ab. Die revolutionäre Hoffnung richtet sich dabei auf die Herkunftsländer. Europa wird primär als Basis wahrgenommen, von der aus revolutionäre Aktivitäten organisiert werden können. Die weltablehnende Variante findet sich in der Regel in mystischen Zirkeln, die sich aus der Gesellschaft zurückziehen und den Kontakt nach Möglichkeit einschränken.

Die antihegemoniale Position wird von den beiden anderen Positionen entschieden kritisiert. Die Kritik aus den Kreisen des individuiert-wertepluralen Islam lautet, dass diese Position unrealistisch sei. Es sei eine Illusion zu meinen, man könne aus der Gesellschaft herausoptieren. Tatsächlich leugnet die antihegemoniale Position, dass das Leben als Muslime in der europäischen Gesellschaft bedeutet, dass sie - wie Stuart Hall es formuliert - »unwiderruflich das Produkt mehrerer ineinander greifender Geschichten und Kulturen sind und zu ein und derselben Zeit mehreren >Heimaten < und nicht nur einer besonderen Heimat angehören« (Hall 1992:310). Dies gilt auch schon deshalb, weil sich Vertreter dieser Positionen ihrer eigenen Tradition mit dem Rüstzeug zuwenden, das sie an europäischen Schulen und Universitäten vermittelt bekommen haben (Kepel 1987; Al Azmeh 1993; Schiffauer 1999; 2000). Dies prägt nachhaltig ihre Sprache und ihren Denkstil. Am deutlichsten wird dies, wenn die Wortwahl und die Thematik ihrer Positionen manchmal bis in die Details die Rhetorik der radikalen Linken aufgreift, wie es bei dem revolutionären Flügel der Fall ist. Aber auch der quietistische Zirkel ist nicht von der weiteren Gesellschaft unbeeinflusst geblieben. Wie Gerdien Jonker jüngst gezeigt hat unterscheidet sich z.B. die Stellung der Frauen in der Süleymanc1-Gemeinde wesentlich von der in traditionalen Gemeinden in der Türkei (Jonker 2002). Die Betonung von kultureller Reinheit und religiösem Absolutismus leugnet die Tatsache, dass sie, wie Hall es formuliert, »unwiderruflich Übersetzer« sind.

Die zweite Kritik aus dem Kreis des werteplural-individuierten Islam betrifft die Tendenz zur sektiererischen Intoleranz vor allem bei den Vertretern der revolutionär antihegemonialen Position. Gerade die Tendenz zur Abgrenzung und Intoleranz erscheint den anderen Muslimen als Widerspruch zu dem Einheitsgebot des Islam und der damit verbundenen prinzipiellen Offenheit. Auf besondere Kritik in diesem Zusammenhang waren die Fetwas gestoßen, in denen Kaplan Muslime, die eine andere Haltung zum Staat einnahmen als er, zu Apostaten erklärte.

Vertreter des kommunalistisch-orthodoxen Islam würden sich dieser Kritik zum Teil anschließen: Sie würden aber darüber hinaus kritisieren, dass die antihegemoniale Politik politisch völlig illusionär sei. Sie wäre nachgerade kontraproduktiv für jede Politik, die versucht, einen Raum für den Islam zu schaffen. Letztendlich würde die antihegemoniale Politik nur den Feinden des Islam in die Hände arbeiten.

Auf diese Kritik antworten die Ultraorthodoxen - etwas hilflos - mit einem Hinweis auf Gottes Willen. Gott kann es doch einfach nicht wollen, dass ein Teil der Gläubigen sich verwestlicht und ein anderer Teil der Gläubigen wichtige Positionen räumt, einfach um sich mit 
den Feinden des Islam gut zu stellen. Man kann also hoffen, dass er diejenigen unterstützt, die seine Offenbarung ernst nehmen.

Das folgende Schema benennt die drei Positionen des Diaspora-Islam der zweiten Generation und führt stichwortartig die Kritik auf:

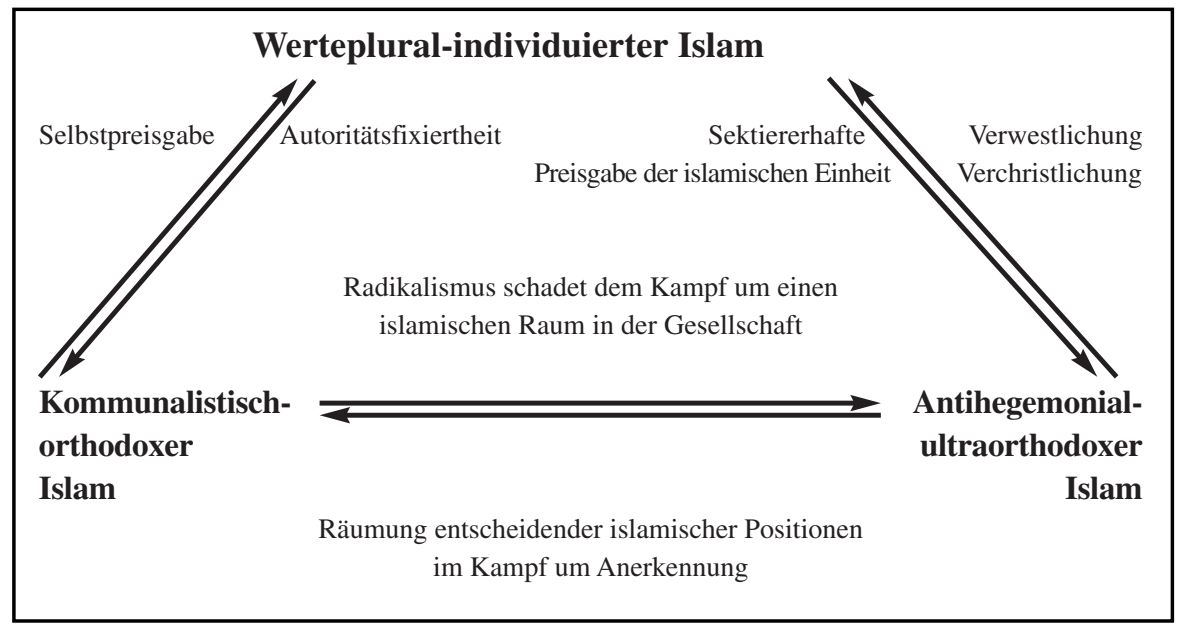

Das Schema führt die drei Positionen auf, die sich in der Diaspora herausbilden. Die Positionen neben den Pfeilen benennen jeweils die zentralen Kritikpunkte aneinander; die Pfeilspitze zeigt auf die Position, an der Kritik geübt wird.

Vertreter des individuierten Islam kritisieren am kommunalistischen Islam den Autoritarismus. Dieser widerspricht ihren Konzeptionen eines »bewussten « Islam, der sich nicht von Autoritäten leiten lässt, sondern durch eine individuelle und kritische Aneignung der Schriften bestimmt ist. Sie kritisieren am antihegemonialen Islam den sektiererischen und darüber hinaus intoleranten Geist, der im Widerspruch zu dem steht, was für sie den Kern des Islam ausmacht, nämlich ein Bekenntnis zu Frieden, Toleranz und Offenheit.

Vertreter des kommunalen Islam kritisieren am individuierten Islam umgekehrt einen »liberalistischen« Individualismus eines »anything goes«. Dies würde letztendlich zu Anpassung, Selbstverlust, Auflösung führen. An der antihegemonial-ultraorthodoxen Position kritisieren sie neben dem Sektierertum vor allem auch eine unrealistische Politikhaltung. Die Politik der Ultraorthodoxie würde letztendlich eine Politik der Verortung in Europa völlig unmöglich machen.

Vertreter der antihegemonial-ultraorthodoxen Position kritisieren am individuierten Islam die »Verwestlichung «. Für sie haben die von diesen Kreisen bezogenen Positionen nichts mehr mit dem Islam zu tun. Das Bekenntnis zu einer privaten Religion entspräche dem Christentum, nicht aber dem Islam. Der kommunalistischen Position konzedieren sie, dass sie zwar im Prinzip gegen diese Auflösungserscheinungen angeht; da sie sich aber der Definitionsmacht von Nicht-Muslimen unterwirft, wird sie früher oder später Positionen einnehmen, die mit dem Islam nichts zu tun haben.

Jede dieser Positionen hat ihre innere Logik - und verstrickt sich in Widersprüche, die von den anderen Positionen benannt werden. Mein Anliegen war es zu zeigen, dass diese Widersprüchlichkeit die Zerrissenheit der Migrationssituation und die Abwertung, die der Islam in Europa erfährt, reflektiert. Die Erfahrung der Macht schreibt sich in diese Formierungen ein. 


\section{Die Rezeption des Diaspora Islam in den Gemeinden}

Die drei Haltungen der zweiten Generation haben (noch) zu keiner eigenen Organisationsform gefunden, wie dies bei den entsprechenden Gemeinden in der jüdischen Diaspora der Fall ist. Vielmehr finden sich Vertreter aller Positionen in allen Gemeinden wieder, die sich in den ersten Jahren der Migration in Europa etabliert haben. In jeder Gemeinde finden sich Personen mit individuierten, kommunalistisch-orthodoxen und antihegemonial-ultraorthodoxen Einstellungen - wenn auch in unterschiedlichem Ausmaß. Die individuierte Haltung findet sich primär in der DİTİB - und zwar wegen des von dieser Gemeinde vertretenen Bekenntnisses zu einem Islam, der die Religion als Privatsache zwischen Mensch und Gott betrachtet. Die kommunalistisch-orthodoxe Position findet sich dagegen vorwiegend in der Milli Görüș. Die radikal antihegemoniale Position wird in ihrer revolutionären Variante im Kalifatsstaat und in ihrer quietistischen von den Süleymancı vertreten. Während also auf der Ebene zwischen den Organisationen nach wie vor die Ausrichtung an der Türkei bestimmend ist, vertreten in den Gemeinden (meist) Angehörige der zweiten Generation Positionen, die in Bezug auf die Aufnahmegesellschaft entwickelt werden. Damit ergibt sich also ein komplexes Webmuster, das auch die Möglichkeiten neuer Koalitionen bietet.

Die Organisationen haben in unterschiedlichem Ausmaß auf diese Verschiebung der religiösen »Nachfrage« reagiert. Die DİTİB, die - was ihre Anhängerschaft betrifft - eigentlich die natürliche Sachwalterin eines liberalen Islam sein könnte, hat bislang versagt. Dies liegt an dem Charakter als staatliche Behörde und der engen Anbindung an den türkischen Staat. Dieser Umstand erschwert eine eigene Entwicklung von Positionen, die auf die Entwicklung in Europa reagieren könnten. Es ist beispielsweise bezeichnend, dass die DİTİB sich im Jahr 2000, als lebhafte Diskussionen um die Etablierung eines islamischen Religionsunterrichts in Berlin stattfanden, außerstande sah, an den Debatten teilzunehmen (Senatsverwaltung 2000:8). In zentralen Fragen - etwa ob der Unterricht auf Türkisch oder auf Deutsch stattfinden sollte - hatte sich die Organisation noch nicht zu einer Meinung durchringen können.

Im Gegensatz dazu hat in der Milli Görüș seit der Mitte der neunziger Jahre ein Führungswechsel stattgefunden. Die leitenden Positionen wurden systematisch mit Angehörigen der zweiten Generation besetzt, die in Europa aufgewachsen waren. Seitdem versucht die Führungsspitze der Milli Görüs die kollektivistisch engagierte Position aufzugreifen und sie zu einem neo-orthodoxen Diaspora-Islam weiter zu entwickeln. Die türkeibezogenen Konflikte, die die Gemeinden in den siebziger und achtziger Jahren entzweiten, werden von der Führungsspitze für überholt erklärt. Die Aufgabe des Tages sei es, dem Islam einen Platz in der europäischen Öffentlichkeit zu schaffen. In diesem Zusammenhang begann die Gemeinde sich durch eine Reihe bemerkenswerter Positionierungen zu profilieren: Sie trat für einen Islamunterricht auf Deutsch ein, startete unter ihren Anhängern eine Kampagne zur Annahme der deutschen Staatsbürgerschaft und verabschiedete Stellungnahmen, in denen die Gläubigen ermutigt werden, ihre Kinder auf deutsche Bildungsinstitutionen, insbesondere Gymnasien, zu schicken. In internen Diskussionen wurde versucht, die Rolle des Islam im säkularen Rechtsstaat, das Verhältnis zum Christentum und die Rolle der Frau im Islam zu bestimmen. Die Differenz des Diaspora- zum Exil-Islam wurde explizit auf den Punkt gebracht, als Mehmet Sabri Erbakan, der zwischen 1999 und 2002 der Organisation vorstand, in seiner Antrittsansprache verkündete, dass man als Muslim in Europa in einer privilegierten Situation sei: 90 Prozent der Muslime lebten schließlich in Situationen staatlicher Oppression, materiellen Elends oder in Kriegsgebieten. Aus der Privilegierung der europäischen Muslime ergebe sich eine Verantwortung gegenüber dem Welt-Islam. Bei der Milli Görüș hat man, was die Entwicklung eines Diaspora-Islam betrifft, einen umgekehrten Eindruck zur Situation bei der DİTİB. Während die DİTİB hinter den Entwicklungen in den Gemeinden hinterher hinkt, setzt sich die Leitung der Milli Görüș an die Spitze dieser Entwick- 
lungen. Zeitweise hatte man den Eindruck, dass die Positionen, die in der Führungsspitze entwickelt wurden, der ersten Generation in der Gemeinde zu weit gingen. All dem korrespondiert eine vorsichtige Lockerung der Beziehung zu der Türkei. ${ }^{23}$

Die antihegemonial-ultraorthodoxe Position revolutionärer Ausprägung findet sich vor allem im »Kalifatsstaat « vertreten, der, obwohl im Dezember 2001 in Deutschland verboten, weiterhin aktiv ist. Der Kalifatsstaat steht für einen islamistischen revolutionären Panislamismus. Der Traum des Gründers Cemaleddin Kaplan war die islamische Revolution in der Türkei, die Wiedererrichtung des Kalifats und darüber die weltweite Wiederherstellung des eigentlichen Islam. In der auf türkisch erscheinenden Verbandszeitschrift »Ümmet-i Muhammed « (»Die Gemeinde Muhammeds«), nach dem Verbot der Gemeinde in Deutschland 2001 in »Asr-i Saadet« (»Das Zeitalter der Glückseligkeit«) umbenannt, wird die weltweite Unterdrückung des Islam unter besonderer Berücksichtigung der Türkei dargestellt. Europa spielt in dieser Zeitung kaum eine Rolle; die Themenstellungen sind vielmehr diejenigen eines globalen Islamismus. Dies ist grundlegend anders bei der auf deutsch erscheinenden Monatszeitschrift D.I.A. (Der Islam als Alternative). Schon die Abfolge der Themenstellungen zeigt die Entwicklung einer antihegemonialen Position in der Auseinandersetzung mit Europa an. Mit »Schutz der fünf Grundwerte«; »Menschen mit Rechten - Menschen ohne Rechte«, »Ausländer«, »Dschihad«, »Globaler Kapitalismus«, »Nationalismus« werden Themen aufgegriffen, die in den europäischen Debatten eine zentrale Rolle spielen. Für die antihegemonial-ultraorthodoxe Position weltflüchtiger Prägung steht die Süleymanc1-Gemeinde. Die Gemeinde ist deshalb interessant, weil es Ende der neunziger Jahre so aussah, als würden sich Vertreter von orthodoxen Positionen durchsetzen und sich die Gemeinde zur Gesellschaft hin öffnen. Diese Entwicklung in der Diaspora-Gemeinde wurde 2000 vom Zentrum in der Türkei abrupt gestoppt (hierzu Jonker 2002)

Wenn in all den drei Gemeinden unter Mitgliedern der zweiten Generation, sagen wir, Positionen eines individuierten Islam existieren, dann heißt dies natürlich nicht, dass diese Positionen deckungsgleich wären: Vielmehr ergeben sich komplexe Synthesen, die zu sehr unterschiedlichen Ausprägungen führen. Wer im Kalifatsstaat liberale Positionen vertritt, wird beispielsweise darauf insistieren, dass man sich die Schriften individuell aneignen und eine Pluralität von Lesarten respektieren muss. Er wird auch Kritik an Tendenzen zu sektenhaften Verkrustungen in der Gemeinde üben. Gleichzeitig wird er aber ein sehr dezidiertes politisiertes Weltbild vertreten und sich in dieser Hinsicht stark von einem Liberalen bei der DİTiB unterscheiden.

\section{Ausblick}

In der Diskussion wird die Entwicklung eines europäischen Islam in der Regel mit der Assimilierung des Islam assoziiert: Der europäische Islam wird »unser« Islam sein: verhandlungsbereit, tolerant und weltoffen. Es war die Absicht des Textes zu zeigen, dass unter den Bedingungen faktischer Diskriminierung eine derartige Entwicklung unwahrscheinlich ist. Auch der indviduierte Islam (der noch die meisten Ähnlichkeiten mit dem Phantasma der Europäer hat) wird angesichts der europäischen Haltung seine Widerständigkeit behalten, auch wenn dieser Widerstand oft wenig sichtbar sein wird, weil er sich in Rückzug äußert. Daneben werden sich kommunalistisch-orthodoxe und antihegemonial-ultraorthodoxe Positionen weiter entfalten. Es gibt nicht den europäischen Islam, sondern eine Vielfalt von Hal-

23) Es bleibt abzusehen, wie diese Entwicklung angesichts der Führungskrise in der Milli Görüș weitergeht. Der charismatische Mehmet Sabri Erbakan trat im Oktober 2002 von seinem Amt zurück, offiziell aus Gesundheitsgründen, nach einem Bericht der Zeitschrift Hürriyet wegen einer Affäre. Sein Nachfolger, Yavuz Celik Karahan, setzt die Linie fort. 
tungen, die sich über die Verortung des Islam in Bezug auf Europa, auf das Herkunftsland und auf den globalen Islam explizit oder implizit auseinander setzen. Es gibt jedoch nicht nur eine Vielzahl von Stimmen, sondern auch eine prozessuale Dynamik. Sie ergibt sich schon daraus, dass jede Position, die in der Suche nach einem Ort in Europa entwickelt wird, ihre Probleme birgt. Diese Probleme sind aber ein Stachel, der die Suche weiter vorantreibt.

Dabei ist in diesem Text auf eine besondere Quelle der religiösen Pluralität in Europa noch nicht eingegangen worden: Wenn hier von »Europa die Rede war, dann ist dies eine problematische Verallgemeinerung. Tatsächlich sind die Migranten ja mit sehr unterschiedlichen politischen Kulturen in Europa konfrontiert. Die Migration hat in allen europäischen Zivilgesellschaften eine Diskussion über ihr Selbstverständnis ausgelöst. Dies hat wiederum sehr unterschiedliche Lösungen gefunden. Während in Großbritannien beispielsweise bewusst versucht wird, »Britishness « neu zu definieren, findet man in Frankreich ein Rückbesinnen auf die Werte des republikanischen Universalismus. Während der Exil-Islam der ersten Generation europaweit sehr ähnliche Positionen entwickelt hatte, weil er Europa insgesamt den Rücken zukehrte, gilt dies für die Positionen einer zweiten Generation nicht mehr. Die Differenzen zwischen liberalen, orthodoxen und ultraorthodoxen Positionen werden sich aller Voraussicht nach in England anders entwickeln als in Frankreich, in den Niederlanden anders als in Deutschland. Die schrittweise Ersetzung des Türkischen als Sprache, in der die Debatten geführt werden, durch die jeweiligen Landessprachen wird die Diversifizierung weiter vorantreiben. Der europäische Islam wird also immer vielstimmiger werden. 


\section{Literatur}

Al-Azmeh, Aziz. 1993. Islams and Modernities. London: Routledge.

Anderson, Benedict (1998). Long Distance Nationalism. In ders. The Spectre of Comparisons. Nationalism, Southeast Asia and the World. London/New York, Verso: 58-74.

Atacan, Fulya. 1993. Kutsal Göç. Radikal Islamcı bir grubun anatomisi. Ankara: Bağlam Yayıncılık.

Berger, Peter L. 1967/1973. Zur Dialektik von Religion und Gesellschaft. Frankfurt am Main: Fischer.

Bhabha, Homi. 1993. Remembering Fanon: Self, psyche and the colonial condition. In Colonial discourse and post-colonial theory. A reader, hrsg. von Williams, Patrick und Laura Chrisman. Hemel Hempstead: Harvester, 112-123.

Boyarin, D. and J. Boyarin (1993). »Diaspora: Generational Ground of Jewish Identity.« Critical Inquiry 19(4): 693-725.

Butler, Judith. 2001/1997. Psyche der Macht. Das Subjekt der Unterwerfung. Gender Studies. Frankfurt am Main. Suhrkamp.

Caeiro, Alexandre. 2003. The European Council for Fatwa and Research. Paper read at Fourth Mediterranean Social and Political Research Meeting, at Florenze - Montecatini Terme.

Çaglar, Ayșe. 2003. Encountering the State in migration driven transnational fields: Turkish immigrants in Europe. Habilitation. FU Berlin.

Clifford, James. 1986. On Ethnographic Allegory. In Writing Culture. The Poetics and Politics of Ethnography, hrsg. von Clifford, James und George E.Marcus. Berkeley: University of California Press, 98121.

Clifford, James 1994. »Diasporas.« Cultural Anthropology 9(3): 302-38.

Cohen, Anthony P. 1985. The Symbolic Construction of Community. London und New York: Routledge.

Dantschke, Claudia: Das Ziel ist die »Islamische Republik Deutschland«www.politikforum.de/forum/archive/6/2004/abgelesen am 1.12.2004

Düttmann, Alexander G. 1997. Zwischen den Kulturen. Spannungen im Kampf um Anerkennung. Frankfurt am Main: Suhrkamp.

Fanon, Frantz. 1981. Die Verdammten dieser Erde. Frankfurt am Main: Suhrkamp.

Foucault, Michel. 1991. Überwachen und Strafen. Die Geburt des Gefängnisses. Frankfurt am Main: Suhrkamp.

Gençel, Aylin. 2003. Images of Islam in the Diaspora. An Ethnographic Study of a Turkish Family in Berlin. Masterarbeit. Fakultät für Kulturwissenschaften, Europa-Universität Viadrina Frankfurt (Oder).

Gilroy, Paul. 1993. Urban Social Movements, $>$ Race $<$ and Community. In Colonial discourse and postcolonial theory. A reader, hrsg. von Williams, Patrick und Laura Chrisman. Hemel Hempstead: Harvester: 404-420.

Goffman, Erving. 1963/1980. Stigma. Frankfurt am Main: Suhrkamp.

Greve, Martin. 2002. Die Musik der imaginären Türkei. Musik und Musikleben im Kontext der Migration aus der Türkei nach Deutschland. Habilitation. Fakultät für Geisteswissenschaften, Technische Universität Berlin.

Habermas, Jürgen. 1992. Anerkennungskämpfe im demokratischen Rechtsstaat. In Multikulturalismus und die Politik der Anerkennung, hrsg. von Taylor, Charles. Frankfurt am Main: Fischer, 147-196.

Hall, Stuart. 1994. Die Frage der kulturellen Identität. In Hall, Stuart: Rassismus und kulturelle Identität. Hamburg: Argument Verlag, 180-222. ORIGINAL.

Honneth, Axel. 1992. Kampf um Anerkennung. Zur moralischen Grammatik sozialer Konflikte. Frankfurt am Main: Suhrkamp.

Jonker, Gerdien. 2002. Eine Wellenlänge zu Gott: Der Verband der islamischen Kulturzentren in Europa. Bielefeld: Transcript.

Kepel, Gilles. 1987. Les Banlieues de l'Islam. Paris: Editions du Seuil.

Klinkhammer, Grit 2000. Moderne Formen islamischer Lebensführung. Marburg, Diagonal. 
Mumcu, Uğur. 1987. Rabita. Istanbul: Tekin.

Roth, Philip 1993. Operation Shylock. A Confession. New York, Simon\&Schuster.

Schiffauer, W. (2000). Die Gottesmänner. Türkische Islamisten in Deutschland. Eine Studie zur Herstellung religiöser Evidenz. Frankfurt am Main, Suhrkamp.

Schiffauer, Werner, Gerd Baumann, Riva Kastoryano und Steven Vertovec (Hrsg). 2002. Staat-SchuleEthnizität. Münster: Waxmann Verlag.

Schiffauer, Werner. 1997. Islam as a Civil Religion: Political Culture and the Organisation of Diversity in Germany. In The Politics of Multiculturalism in the New Europe, hrsg. von Modood, Tariq und Pnina Werbner. London und New York: Zed Books.

Schiffauer, Werner. 1988. Migration and Religiousness. In The New Islamic Presence in Western Europe, hrsg. von Gerholm, Lithman. London: Mansell Publ. Lim.

Schiffauer, Werner. 1997.Islamic Vision and Social Reality. The Political Culture of Sunni Turks Muslim in Germany. In Islam in Europe - The Politics of Religion and Community, hrsg. von Vertovec, Steven und C. Peach. London: McMillan.

Schiffauer, Werner. 1999. Islamism in the diaspora. The fascination of political Islam among second generation German Turks. Oxford. Transnational Communities Programme - Working Paper Series. http://www.transcomm.ox.ac.uk

Schiffauer, W. 2004. Die Islamische Gemeinschaft Milli Görüș - ein Lehrstück zum verwickelten Zusammenhang von Migration, Religion und sozialer Integration. Migrationsreport 2004. Fakten - Analysen - Perspektiven. K. J. Bade, M. Bommes and R. Münz. Frankfurt; New York, Campus Verlag: 67 - 96.

Senatsverwaltung für Schule, Jugend und Sport (Hrsg). 2000. Islamischer Religionsunterricht an Berliner Schulen. Probleme, Fragen, Antworten. Podiumsdiskussion am 29.3.2000 in der Friedrich-Ebert-Stiftung. Berlin.

Sivan, Emmanuel 1985. Radical Islam. Medieval Theology and Modern Politics. New Haven and London, Yale University Press.

Taylor, Charles. 1992. Die Politik der Anerkennung. In Taylor, Charles: Multikulturalismus und die Politik der Anerkennung. Frankfurt am Main: Fischer,13-78.

Vertovec, Steven. 2002. Religion in Migration, Diasporas and Transnationalism. Vancouver Centre of Excellence. Research on Migration and Integration in the Metropolis. Working Paper Series No.02-07.

Prof. Dr. Werner Schiffauer

Europa-Universität Viadrina

Postfach 775

15207 Frankfurt (Oder)

schiffauer@euv-frankfurt-o.de 